\title{
Fast Atomic-Scale Chemical Imaging by STEM EDS for Study of Crystalline Materials and Dynamic Phase Transformations
}

\author{
Ping Lu ${ }^{1}$, Ren Liang Yuan ${ }^{2}$ and Jian Min $\mathrm{Zuo}^{2}$ \\ 1. Sandia National Laboratories, Albuquerque, NM USA \\ 2. Department of Materials Science and Engineering, University of Illinois at Urbana-Champaign, 1304 \\ W Green St, Urbana, IL USA
}

Chemical imaging at the atomic-scale provides a useful tool to investigate material structures and has been recently demonstrated in aberration corrected scanning transmission electron microscopy (STEM). By scanning an angstrom-sized electron probe across a crystal sample aligned along a low-axis orientation and collecting either electron energy-loss spectra (EELS) or energy-dispersive x-ray spectra (EDS), atomic-scale chemical imaging has been achieved. Atomic-scale STEM EDS chemical imaging offers a benefit of easy interpretation with a one-to-one correspondence between image and structure, however has a severe shortcoming due to the poor efficiency of $\mathrm{x}$-ray generation and collection. To compensate the poor efficiency, spectral imaging STEM EDS datasets are often acquired as a series of frames, where the same region is scanned multiple times (typically over few 100 times) and spatially drift-corrected to build a dataset [1,2]. A typical data collection requires more than a few 100 seconds, subjecting the samples to high irradiation of electrons. This limits the materials that can be studied by the technique to only those that are electron radiation-resistant, and prevents the study of phase transformations and dynamics, which requires the atomic-scale chemical structural information resolved on a much shorter time scale.

In this study, we report a novel approach that allows realization of rapid atomic-scale chemical imaging by STEM EDS. Using $\mathrm{LaAlO}_{3}$ (LAO) as a model crystal, we find averaged atomic-scale chemical maps for $\mathrm{La}$ and $\mathrm{Al}$ atoms can be extracted from an area of $\sim$ few $10 \mathrm{~nm}^{2}$ acquired with the acquisition time of $\sim 2$ seconds or less.

Figure 1 illustrates the method. The x-ray counts, represented by red dots, are distributed on a tile pattern which represents a crystal lattice that gives rise to the x-rays when electron probe is scanned over the region (Fig.1a). Under the atomic-scale STEM EDS imaging conditions, the $x$-ray counts, which might be seemly random and sparsely scattered under a short acquisition time (i.e., less than few seconds), are not really random but statistically localized to the atomic columns. The statistical distribution or chemical map (Fig.1c) can be obtained by translating the X-rays distributed over the large region to the equivalent pixel positions within a single unit cell via lattice-vector translation, as illustrated in Figs.1a and 1b. By concentrating x-ray signals into the unit cell, an acquisition time savings of over 100 times can be achieved, and an averaged chemical map can be obtained with the acquisition time of less than 1 sec.

Figure 2a shows experimental x-ray maps from a LAO crystal in [100] which is acquired with a total acquisition time of about $1.8 \mathrm{sec}$ from a region of $4.46 \mathrm{~nm} \times 4.46 \mathrm{~nm}$. The maps are overlaid on the HAADF image acquired simultaneously. Using the lattice-vector translation method as illustrated in Fig.1, chemical maps for Sr and Ti can be obtained (Figs.2b and 2c), respectively, and the RGB map obtained (Fig.2d, Sr in red, Ti in green) clearly shows the structure of the LAO (Fig.2e). We have since applied the method to study several different crystals such as $\mathrm{SrTiO}_{3}$, and $\mathrm{GaN}$, and have verified the 
technique using statistically based phenomenological modeling [3]. The fast atomic-scale chemical mapping has also been applied to study the electron-beam induced phase transformation in a layered lithium transition-metal oxide, $\mathrm{Li}\left[\mathrm{Li}{ }_{0.2} \mathrm{Ni}_{0.2} \mathrm{Mn}_{0.6}\right] \mathrm{O}_{2}$ (LNMO). By capturing a series of time-resolved chemical maps, a new kinetic mechanism for the LNMO phase transformation has been determined [3, 4].

References:

[1] Lu, P. et al, Sci. Rep. 4, 3945 (2014).

[2] Lu, P. et al, Microsc. Microanal 20, 1782-1790 (2014).

[3] Lu, P. et al, submitted to Nano Letters.

[4] Sandia National Laboratories is a multi-program laboratory managed and operated by Sandia Corporation, a wholly owned subsidiary of Lockheed Martin Corporation, for the US Department of Energy's National Nuclear Security Administration under contract DE-AC04-94AL85000.

1

1 a

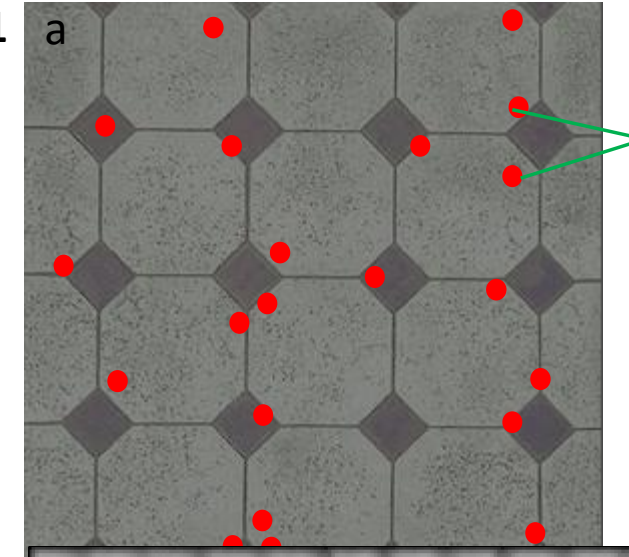

2

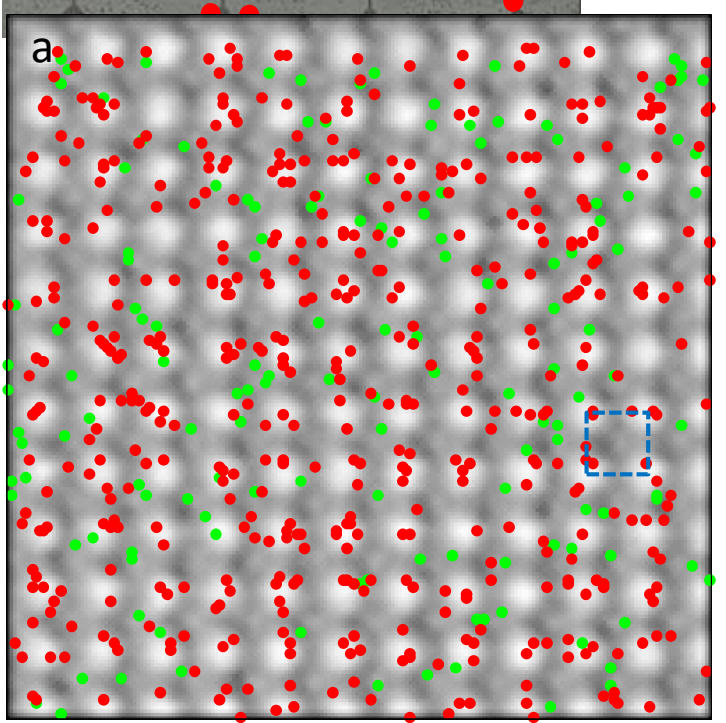

b Unit cell

C

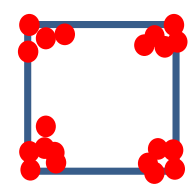

Figure 1. Schematic showing the lattice-vector translation of x-rays (red-dots) distributed over a crystal region (represented by the tile pattern in (a)) to their equivalent positons within the unit cell (b), and formation of a chemical map (c). Only 20 x-rays are used in this illustration. It shows that few $\mathrm{x}$-rays are needed to form a recognizable map, which can be achieved by STEM EDS with a collection time of less than 1 sec.
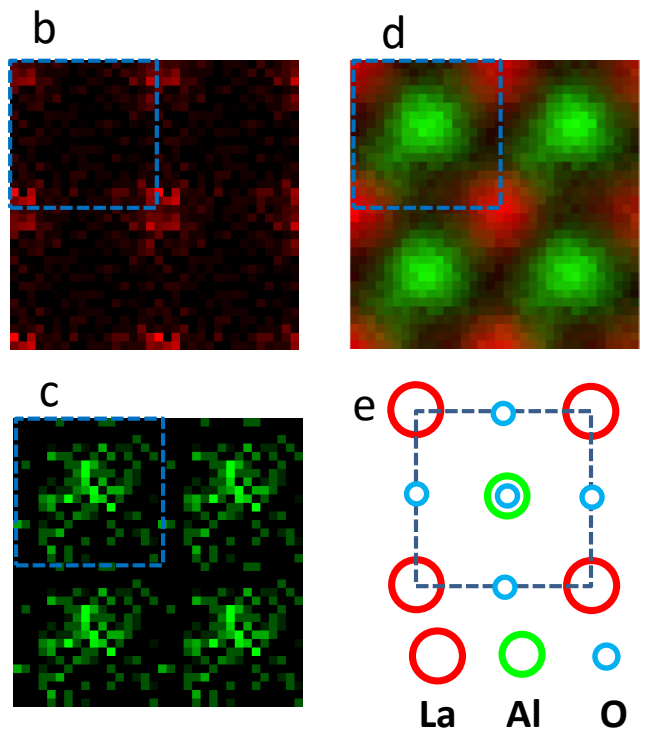

Figure 2. (a) raw x-ray count maps of La L(red-dot) and Al K (green-dot), obtained experimentally by STEM EDS with a total acquisition of $1.8 \mathrm{sec}$, overlaid on the reference HAADF image acquired simultaneously; (b, c) x-ray count maps ( $2 \times 2$ unit-cells shown) for La L, and Al K x-rays, obtained by the lattice-vector translation method using the x-ray maps in (a); (d) (La, Al) RGB map obtained with the maps in (b) and (c) after 3-point smoothing; and (e) projected LAO structure in [001] direction. A FEI Titan ${ }^{\mathrm{TM}}$ G2 80-200 STEM with a Cs probe corrector and ChemiSTEM ${ }^{\mathrm{TM}}$ technology operated at $200 \mathrm{kV}$ was used in this study. 